\title{
Regulation of E-cadherin
}

\author{
Z. Yang*, H. Zhang*, R. Kumar \\ Department of Molecular and Cellular Oncology, The University of Texas M. D. Anderson Cancer Center, \\ Houston, Texas, USA.
}

\begin{abstract}
Numerous studies suggest that loss of E-cadherin is necessary to induce Epithelial-mesenchymal transition (EMT) and metastasis. Snail is a major contributor to EMTs. The Snail family of zinc-finger transcription factors interact with the E-cadherin promoter to repress transcription during EMT. The present article reviews the regulation of E-cadherin and discusses recent novel insights into the molecular basis in the process of EMT.
\end{abstract}

Keywords: E-cadherin; Snail; Epithelial-mesenchymal transition

E-cadherin (uvomorrulin), the founding member of the cadherin superfamily of calcium-dependent, transmembrane glycoproteins, plays a critical role in establishing adherens-type junctions. E-cadherin contains a prototypic extracellular domain that mediates homophilic protein-protein interactions in a zipperlike fashion. The intracellular domains of cadherins interact with several proteins collectively known as catenins. The resulting molecular complex binds to $\alpha$-catenin and assembles other peripheral cytoplasmic proteins to connect E-cadherin to the actin cytoskeleton. E-cadherin plays an essential role in normal physiologic processes such as development, cell polarity, and tissue morphology [1], and in pathologic states such as epithelial-mesenchymal transition (EMT), a process usually accompanied by tumor dedifferentiation, infiltration, and metastasis. Alterations in E-cadherin expression or functions are common during carcinogenesis. In general, aberrant spatial-temporal expression or repression of $\mathrm{E}$-cadherin is accompanied during EMT, an essential component of cancer progression to more aggressive

\footnotetext{
*Equal contribution.
}

Correspondence to: Rakesh Kumar, Department of Molecular and Cellular Oncology, The University of Texas M. D. Anderson Cancer Center, Houston, Texas, USA. E-mail: rkumar@mdanderson.org

Publication date 31/03/05

BCO/315/2004/FO phenotypes. In contrast, restoration of E-cadherin expression enhances intercellular adhesion, inhibits tumorigenicity, and suppresses the invasiveness of epithelial tumor cells $[2,3]$. Due to the loss of cell-tocell junctions during EMT, E-cadherin loss leads to an increased pool of the cytoplasmic $\beta$-catenin as well as its transcription activity, resulting in the stimulation of $\beta$-catenin-responsive growth-regulatory genes [2]. Here we will briefly summarize the regulation of E-cadherin expression and discuss novel insights by which upstream regulators of E-cadherin might control the process of EMT, with a particular emphasis in breast cancer cells.

\section{Genetic control of E-cadherin expression}

The E-cadherin encoding gene, $\mathrm{CDH} 1$, maps to a region on chromosome 16q22.1, a region frequently associated with the loss of heterozygosity in sporadic breast cancers [4]. Since somatic mutations inactivating the $\mathrm{CDH} 1$ gene are found in over $50 \%$ of diffuse-type gastric and infiltrative lobular breast cancers [5], E-cadherin has been proposed to have a causal role in some human cancers. However, the finding that E-cadherin mutations are rare in ductal breast cancers [4] suggests the potential involvement of epigenetic modifications in controlling the functions of E-cadherin. 


\section{Epigenetic control of E-cadherin inactivation}

Epigenetic mechanisms such as hypermethylation of the E-cadherin promoter [6,7], Histone $\mathrm{H} 3$ deacetylation (HDAC) in the context of CpG-methylationmediated gene silencing [8], and transcriptional silencing have all been linked to the inactivation of E-cadherin expression. Analysis of somatic cell hybrids of E-cadherin-positive and -negative breast cancer cells suggests that the loss of E-cadherin expression in some breast cancers may be linked with a dominant repression transacting pathway [9], presumably due to dysregulation of transcription factors, support the notion of direct transcriptional silencing of E-cadherin as a major regulatory mechanism in human cancers.

\section{Transcriptional controls of E-cadherin}

The transcription of E-cadherin is controlled by both positive- and negative-regulatory elements located in its $5 \mathrm{~V}$ promoter region $[3,10-12]$. The $\mathrm{V}$ promoter region also contains three E-box motifs, and proteinbinding motifs with a core consensus sequence of CANNTG [3,13-17], which have both negative- and positive-regulatory functions. The E-box motifs function as negative regulators of E-cadherin in mesenchymal and in transformed cells by binding to Snail [3,13-17] or Zeb [13]. In addition, the E-box motifs could also impart a positive-regulatory function in epithelial cells [12] due to interactions with bHLH transcription factors [14].

The demonstration that the Snail family of zincfinger transcriptional repressors control E-cadherin expression in epithelial cells has opened a new avenue of research in the field EMT. Examples of zinc-finger transcription repressors of E-cadherin include: Snail, Slug, ZEB1 and SIP1 (ZEB2) [17], and Twist [18]. Two widely studied repressors of E-cadherin expression and consequently, of EMT, are Snail and Slug $[3,14,15]$, with ectopic expression of Snail or Slug in epithelial cells downregulating E-cadherin and promoting fibroblastic, tumorigenic and invasive characteristics $[3,14,15]$. Snail also activates the transcription of vimentin and fibronectin, which are bona fide markers of mesenchymal differentiation. Accordingly, carcinoma cells with low or no E-cadherin contain high levels of Snail-1 $[3,14,19,20]$. In addition to cancer, Snail is also critical during developmental processes, as Snail-1 knockout mice were unable to undergo gastrulation and neural-crest EMT [16], and is embryonic lethal due to its inability to downregulate E-cadherin and undergo EMT [17].

Slug was first identified as another repressor of E-cadherin in chicken, where it was shown to be critical in the induction of EMT during embryonic development [21]. However, this function is not conserved in all vertebrates, as its expression does not correlate with the loss of E-cadherin expression or EMT during mouse embryogenesis [3,22], and unlike Snail, Slug-null mice are viable and fertile [22]. Interestingly, the status of Slug expression correlates well with the loss of E-cadherin in human breast carcinomas [15]. There is also evidence to suggest that Slug might participate in EMT via downregulation of the components of the desmosome adhesion complex [23], in addition to downregulation of E-cadherin in some epithelial cell lines in a specific cellular contexts.

Transcriptional repressor activity of Snail has been linked with the $\mathrm{C}$-terminus zinc fingers which mediate the sequence specific DNA binding to the E-box consensus sequence of CANNTG $[3,14,19,23-28]$. It is hypothesized that the repressor function of the Snail proteins is partially mediated via its competition with the bHLH transcription factors to bind the E-box motif $[16,26]$. Although the repressor activity depends greatly on the zinc-finger region, at least two other regions in the $\mathrm{N}$-terminus are important for repressor function. The SNAG (Snail/Gfi) domain is conserved in the N-terminal region of all vertebrate Snail genes, and shown to be important for the repressor function of the Snail proteins in mammalian cells. Several vertebrate Snail family members also contain a partial CtBP interaction domain (CID) consensus sequence. The transcriptional repressor role of the Snail family of proteins is therefore mediated by its ability to compete for regulatory elements in the E-cadherin promoter, the SNAG domain, its interaction with CtBP, or a combination of the all three routes.

\section{Cooperation of transcriptional repression with epigenetic modification}

One widely accepted mechanism by which Snail represses the E-cadherin promoter includes the recruitment of repressor complexes involving Sin3A, or HDACs [9]. In addition, E-cadherin transcription is shown to be silenced by a CtBP corepressor complex containing different methylase and HDAC activities, as well as EF1 and SIP1 [19]. The complexity of repression of E-cadherin is further evident by the recent finding that the Snail gene is directly inhibited, in an HDAC-dependent manner, by MTA3, an estrogen-dependent component of the large transcriptional corepressor complex Mi-2/NuRD [29-31]. In addition, corepressor function of Snail is also compromised by its subcellular relocalization to the cytoplasm $[31,32]$. Overall, these findings indicate that Snail regulation of E-cadherin might be regulated by the dynamic interplay among multiple coregulators in a temporal and spatial manner. 


\section{Signaling control of E-cadherin expression}

The process of EMT as well as E-cadherin expression could be influenced by a variety of polypeptide growth factors and growth-factor-responsive signaling pathways. However, mechanisms by which signaling pathways regulates the expression of E-cadherin are poorly understood, but widely believed to involve modulation of the phosphorylation and/or the steady-state level of Snail [23]. For example, GSK$3 \beta$-mediated phosphorylation of Snail (at motif 2) negatively controls the stability of Snail protein, and thus, could lead to re-expression of E-cadherin in cells [32]. In contrast, stimulation of p21-activated kinase 1, a major signaling nodule downstream of growth factors and the Small GTPases, is required for an optimum transcription repression activity of Snail [33]. The underlying mechanism of Pak1 regulation of Snail activity involves Pak1 phosphorylation of Snail on serine 246 and its accumulation in the nucleus to exert its repressor functions [33]. Therefore, the corepressor functions of Snail and consequently, the process of EMT, could be modulated in both a negative and a positive manner depending on the nature of the signaling kinase activated or posttranslational modification of Snail.

\section{Future direction}

Regulation of E-cadherin plays a crucial role in EMT and tumor progression. A hierarchy of different mechanisms at multiple levels, including genetic, epigenetic, and transcriptional regulations, may finally define the E-cadherin activity in a dynamic, as well as a cell and tissue specific manner. Since most of our current understanding of EMT in cancer cells is derived from tissue-culture model systems, it will be important to start combining these approaches with whole animal models as well as with human tumor specimens, to gain a comprehensive view of upstream regulators of E-cadherin that may be important in tumor invasion.

\section{References}

1. Takeichi M. Morphogenetic roles of classic cadherins. Curr Opin Cell Biol 1995; 7: 619-627.

2. Perl AK, Wilgenbus P, Dahl U, Semb H, Christofori G. A causal role for E-cadherin in the transition from adenoma to carcinoma. Nature 1998; 392: 190-193.

3. Cano A, Perez-Moreno MA, Rodrigo I, et al. The transcription factor snail controls epithelial-mesenchymal transitions by repressing E-cadherin expression. Nat Cell Biol 2000; 2: 76-83.

4. Berx G, Van Roy F. The E-cadherin/catenin complex: an important gatekeeper in breast cancer tumorigenesis and malignant progression. Breast Cancer Res 2001; 3: 289-293.

5. Hajra KM, Fearon ER. Cadherin and catenin alterations in human cancer. Gene Chromosom Cancer 2002; 34: 255-268.

6. Strathdee G. Epigenetic versus genetic alterations in the inactivation of E-cadherin. Semin Cancer Biol 2002; 12: 373-379.

7. Grady WM, Willis J, Guilford PJ, et al. Methylation of the $\mathrm{CDH} 1$ promoter as the second genetic hit in hereditary diffuse gastric cancer. Nat Genet 2000; 26: 16-17.

8. Koizume S, Tachibana K, Sekiya T, Hirohashi S, Shiraishi M. Heterogeneity in the modification and involvement of chromatin components of the $\mathrm{CpG}$ island of the silenced human $\mathrm{CDH} 1$ gene in cancer cells. Nucleic Acids Res 2002; 30: 4770-4780.

9. Hajra KM, Ji X, Fearon ER. Extinction of E-cadherin expression in breast cancer via a dominant repression pathway acting on proximal promoter elements. Oncogene 1999; 18: 7274-7279.

10. Behrens J, Lowrick O, Klein-Hitpass L, Birchmeier W. The E-cadherin promoter: functional analysis of a GC-rich region and an epithelial cell-specific palindromic regulatory element. Proc Natl Acad Sci USA 1991; 88: 1495-1499.

11. Ringwald M, Baribault $H$, Schmidt $C$, Kemler R. The structure of the gene coding for the mouse cell adhesion molecule uvomorulin. Nucleic Acids Res 1991; 19: 6533-6539.

12. Giroldi LA, Bringuier PP, de Weijert M, Jansen C, van Bokhoven A, Schalken JA. Role of E boxes in the repression of E-cadherin expression. Biochem Biophys Res Commun 1997; 241: 453-458.

13. Guaita S, Puig I, Franci C, et al. Snail induction of epithelialto-mesenchymal transition in tumor cells is accompanied by MUC-1 repression and ZEB1 expression. J Biol Chem 2002; 277: 39209-39216.

14. Batlle E, Sancho E, Franci C, et al. The transcription factor snail is a repressor of E-cadherin gene expression in epithelial tumour cells. Nat Cell Biol 2000; 2: 84-89.

15. Hajra KM, Chen DY, Fearon ER. The SLUG zinc-finger protein represses $\mathrm{E}$-cadherin in breast cancer. Cancer Res 2002; 62: 1613-1618.

16. Perez-Moreno MA, Locascio A, Rodrigo I, et al. A new role for E12/E47 in the repression of E-cadherin expression and epithelial-mesenchymal transitions. J Biol Chem 2001; 276: 27424-27431.

17. Carver EA, Jiang R, Lan Y, Oram KF, Gridley T. The mouse snail gene encodes a key regulator of the epithelialmesenchymal transition. Mol Cell Biol 2001; 21: 8184-8188.

18. Yang J, Mani SA, Donaher JL, et al. Twist, a master regulator of morphogenesis, plays an essential role in tumor metastasis. Cell 2004; 117: 927-939.

19. Nieto MA. The snail superfamily of zinc-finger transcription factors. Nat Rev Mol Cell Biol 2002; 3: 155-166.

20. Jiao W, Miyazaki K, Kitajima Y. Inverse correlation between E-cadherin and Snail expression in hepatocellular carcinoma cell lines in vitro and in vivo. Br J Cancer 2002; 86: 98-101.

21. Nieto MA, Sargent MG, Wilkinson DG, Cooke J. Control of cell behavior during vertebrate development by Slug, a zinc finger gene. Science 1994; 264: 835-839.

22. Jiang $R$, Lan $Y$, Norton CR, Sundberg JP, Gridley T. The Slug gene is not essential for mesoderm or 
neural crest development in mice. Dev Biol 1998; 198 : 277-285.

23. LaBonne C, Bronner-Fraser M. Snail-related transcriptional repressors are required in Xenopus for both the induction of the neural crest and its subsequent migration. Dev Biol 2000; 221: 195-205.

24. Fuse N, Hirose S, Hayashi S. Diploidy of Drosophila imaginal cells is maintained by a transcriptional repressor encoded by escargot. Genes Dev 1994; 8: 2270-2281.

25. Inukai T, Inoue A, Kurosawa H, et al. SLUG, a ces-1-related zinc finger transcription factor gene with antiapoptotic activity, is a downstream target of the E2A-HLF oncoprotein. Mol Cell 1999; 4: 343-352.

26. Kataoka H, Murayama T, Yokode $M$, et al. A novel snailrelated transcription factor Smuc regulates basic helixloop-helix transcription factor activities via specific E-box motifs. Nucleic Acids Res 2000; 28: 626-633.

27. Fujiwara S, Corbo JC, Levine M. The snail repressor establishes a muscle/notochord boundary in the Ciona embryo. Development 1998; 125: 2511-2520.
28. Hemavathy K, Guru SC, Harris J, Chen JD, Ip YT. Human Slug is a repressor that localizes to sites of active transcription. Mol Cell Biol 2000; 20: 5087-5095.

29. Fujita N, Jaye DL, Kajita M, et al. MTA3, a Mi-2/NuRD complex subunit, regulates an invasive growth pathway in breast cancer. Cell 2003; 113: 207-219.

30. Kumar R. Another tie that binds the MTA family to breast cancer. Cell 2003; 113: 142-143.

31. Mishra SK, Talukder AH, Gururaj AE, et al. Upstream determinants of estrogen receptor-alpha regulation of metastatic tumor antigen 3 pathway. J Biol Chem 2004; 279: 32709-32715.

32. Zhou BP, Deng J, Xia W, et al. Dual regulation of Snail by GSK-3beta-mediated phosphorylation in control of epithelial-mesenchymal transition. Nat Cell Biol 2004; 6: 931-940.

33. Yang Z, Rayala S, Nguyen D, et al. Pak1 phosphorylation of Snail, a master regulator of epithelial-to-mesenchyme transition, modulates Snail's subcellular localization and functions. Cancer Res 2005; 65(8) (in press). 